\title{
Random-phase Approximation Treatment Of Edge Magnetoplasmons: Edge-state Screening And Nonlocality
}

\author{
O. G. Balev* and P. Vasilopoulos ${ }^{\dagger}$ \\ * Institute of Physics of Semiconductors, National \\ Academy of Sciences, \\ 45 Pr. Nauky, Kiev 252650, Ukraine \\ ${ }^{\dagger}$ Concordia University, Department of Physics, \\ 1455 de \\ Maisonneuve Blvd O, Montréal, Québec, Canada, H3G 1 M8
}

(July 6, 1998)

\begin{abstract}
A random-phase approximation (RPA) treatment of edge magnetoplasmons (EMP) is presented for strong magnetic fields, low temperatures, and integer filling factors $\nu$. It is valid for negligible dissipation and lateral confining potentials smooth on the scale of the magnetic length $\ell_{0}$ but sufficiently steep that the Landau level (LL) flattening can be neglected. LL coupling, screening by edge states, and nonlocal contributions to the current density are taken into account. In addition to the fundamental mode with typical dispersion relation $\omega \sim q_{x} \ln \left(q_{x}\right)$, fundamental modes with acoustic dispersion relation $\omega \sim q_{x}$ are obtained for $\nu>2$. For $\nu=1,2$ a dipole mode exists, with dispersion relation $\omega \sim q_{x}^{3}$, that is directly related to nonlocal responses.
\end{abstract}

PACS 73.20.Dx, 73.40.Hm

\section{INTRODUCTION.}

Edge magnetoplasmons (EMP's) have atracted considerable interest in the past years [1]- 11] and Ref. [1] contains a discussion of older studies. In [11] we have treated EMPs, $\propto A\left(\omega, q_{x}, y\right) \exp \left[-i\left(\omega t-q_{x} x\right)\right]$, for $\nu=1,2$ and very low temperatures when the unperturbed density profile drops sharply at the edge on a length of the order of the magnetic length $\ell_{0}$. Such a profile is valid for $k_{B} T \ll \hbar v_{g} / \ell_{0}$, where $v_{g}$ is the group velocity of the edge states. Then for $\nu=1,2$ the unperturbed electron density $n_{0}(y)$, normalized to the bulk value $n_{0}$, is calculated as $n_{0}(y) / n_{0}=\left\{1+\Phi\left[\left(y_{r e}-y\right) / \ell_{0}\right]\right\} / 2$, where $y_{r e}$ is the coordinate of the right edge and $\Phi(y)$ the probability integral.

The main results of Ref. [11] were the existence of many symmetric and antisymmetric modes with respect to the edge, some of which can propagate nearly undamped even when the dissipation is strong and were, therefore, termed edge helicons. In this quasi-microscopic treatment, however, the contributions to the current density $j_{\mu}\left(\omega, q_{x}, y\right)$ were obtained from microscopic expressions valid when the components of the electric field $\mathbf{E}$, associated with the wave, are smooth on the $\ell_{0}$ scale. This is not well justified. In Ref. [11] we neglected possible nonlocal effects and approximated the contributions to $j_{\mu}\left(\omega, q_{x}, y\right)$ with those obtained when $E_{y}(y)$ is smooth on the scale of $\ell_{0}$. The model [11] also neglects the screening of the two-dimensional electron gas (2DEG). Here, using a RPA framework we study the effect of nonlocality and of edge-states screening on EMPs for filling factors $6 \geq \nu \geq 1$. For even filling factors $\nu$ we neglect the spin splitting. As we will show, the existence of additional modes for $\nu=1(2)$, predicted in Ref. [11], is confirmed by the present fully microscopic treatment and the results for the fundamental mode remain valid for weak dissipation despite the neglect of screening and nonlocality in [11].

In Sec. II we present the channel-edge characteristics and derive an integral equation for the inhomogeneous wave charge density within a RPA framework. In Sec. III we obtain the fundamental and dipole EMP's for $\nu=1,2$ and in Sec. IV we consider fundamental EMPs for $\nu=4$ and $\nu=6$. Finally, in Sec. V we discuss briefly the results and make concluding remarks.

\section{BASIC RELATIONS}

\section{A. Channel-edge characteristics}

We consider a zero-thickness 2DEG of width $W$ and of length $L_{x}=L$ in the presence of a strong magnetic field $B$ along the $z$ axis. We take the confining potential flat in the interior of the 2DEG $\left(V_{y}=0\right)$ and parabolic at its edges, $V_{y}=m^{*} \Omega^{2}\left(y-y_{r}\right)^{2} / 2, y \geq y_{r} . V_{y}$ is assumed smooth on the scale of $\ell_{0}=\left(\hbar / m^{*} \omega_{c}\right)^{1 / 2}$ such that $\Omega \ll \omega_{c}$, where $\omega_{c}=|e| B / m^{*}$ is the cyclotron frequency $(e<0)$. In the Landau gauge for the vector potential $\mathbf{A}=(-B y, 0,0)$ the one-electron Hamiltonian $\hat{h}^{0}$ is given by 


$$
\hat{h}^{0}=\left[\left(\hat{p}_{x}+e B y\right)^{2}+\hat{p}_{y}^{2}\right] / 2 m^{*}+V_{y},
$$

where $\hat{\mathbf{p}}$ is the momentum operator. The eigenvalues and eigenfunctions corresponding to Eq. (11) near the right edge of the channel, with $y_{0} \equiv y_{0}\left(k_{x}\right)=\ell_{0}^{2} k_{x} \geq y_{r}$, are well approximated by

$$
E_{\alpha} \equiv E_{n}\left(k_{x}\right)=(n+1 / 2) \hbar \omega_{c}+m^{*} \Omega^{2}\left(y_{0}-y_{r}\right)^{2} / 2,
$$

and

$$
\mid \alpha>\equiv \psi_{\alpha}(\mathbf{r})=e^{i k_{x} x} \Psi_{n}\left(y-y_{0}\right) / \sqrt{L},
$$

respectively. Here $\mathbf{r}=\{x, y\}, \alpha \equiv\left\{n, k_{x}\right\}, \Psi_{n}(y)$ is a harmonic oscillator function. The energy spectrum (2) of the n-th LL leads to the group velocity of the edge states $v_{g n}=\partial E_{n}\left(k_{r}+k_{e}^{(n)}\right) / \hbar \partial k_{x}=\hbar \Omega^{2} k_{e}^{(n)} / m^{*} \omega_{c}^{2}$ with characteristic wave vector $k_{e}^{(n)}=\left(\omega_{c} / \hbar \Omega\right) \sqrt{2 m^{*} \Delta_{F n}}, \Delta_{F n}=E_{F}-(n+1 / 2) \hbar \omega_{c}$, and $E_{F}$ is the Fermi energy. The edge of the n-th LL is denoted by $y_{r n}=y_{r}+\ell_{0}^{2} k_{e}^{(n)}=\ell_{0}^{2} k_{r n}$, where $k_{r n}=k_{r}+k_{e}^{(n)}$, and $W=2 y_{r 0}$. We can also write $v_{g n}=E_{e n} / B$, where $E_{e n}=\Omega \sqrt{2 m^{*} \Delta_{F n}} /|e|$ is the electric field associated with the confining potential $V_{y}$ at $y_{r n}$. We also introduce the wave vector $k_{r}=y_{r} / \ell_{0}^{2}$.

For definiteness, we take the background dielectric constant $\epsilon$ to be spatially homogeneous. Assuming $\left|q_{x}\right| W \gg 1$, we can consider an EMP along the right edge of the channel of the form $A\left(\omega, q_{x}, y\right) \exp \left[-i\left(\omega t-q_{x} x\right)\right]$ totally independent of the left edge. For $\nu$ even the spin-splitting is neglected.

\section{B. Wave charge density and electric potential at the channel edge}

As in Refs. [11] and [12], we assume that without interaction the one-electron density matrix $\hat{\rho}^{(0)}$ is diagonal, i.e., $\left.<\alpha\left|\hat{\rho}^{(0)}\right| \beta\right\rangle=f_{\alpha} \delta_{\alpha \beta}$, where $f_{\alpha}=1 /\left[1+\exp \left(\left(E_{\alpha}-E_{F}\right) / k_{B} T\right)\right]$ is the Fermi-Dirac function.

For the application of the RPA we follow the self-consistent field approach of Ref. [13] and references cited therein. The one-electron Hamiltonian in the presence of a self-consistent wave potential $V(x, y, t)=V\left(\omega_{0}, q_{x}, y\right) \exp \left[-i\left(\omega_{0} t-\right.\right.$ $\left.\left.q_{x} x\right)\right]+$ c.c., is $\hat{H}(t)=\hat{h}^{0}+V(x, y, t)$. The corresponding equation of motion for the one-electron density matrix $\hat{\rho}$ reads

$$
i \hbar \frac{\partial \hat{\rho}}{\partial t}=[\hat{H}(t), \hat{\rho}]-\frac{i \hbar}{\tau}\left(\hat{\rho}-\hat{\rho}^{(0)}\right),
$$

where [,] denotes the commutator. On the right hand side (RHS) of Eq. (4) we have introduced the phenomenological infinitesimal term $\propto 1 / \tau(\tau \rightarrow \infty)$ that leads to the correct rules for contour integration around the singularities, cf. Refs. [13] and [14. Notice that $\tau \rightarrow \infty$ corresponds to the collisionless case while using a finite $\tau$ provides the possibility of estimating roughly the influence of collisions. Here the most effective collisions are related to intra-LL and intra-edge transitions, cf. Refs. [11]- [12].

We take the Laplace trasform, with respect to the time t, of Eq. (㺼), write $\hat{R}(\omega)=\int_{0}^{\infty} e^{i \omega t} \hat{\rho} d t$ and $R_{\alpha \beta}(\omega)=<$ $\alpha|\hat{R}(\omega)| \beta>$, and look for its solution in a power series in $V$

$$
R_{\alpha \beta}(\omega)=\sum_{n=0}^{\infty} R_{\alpha \beta}^{(n)}(\omega)
$$

where $R_{\alpha \beta}^{(0)}(\omega)=\left(i f_{\alpha} / \omega\right) \delta_{\alpha \beta}$. Because we consider linear EMPs, it is sufficient to take into account only the first two terms $n=0$ and $n=1$ on the RHS of Eq. (5). Then in $V(x, y, t)$ we can consider only the term $V\left(\omega_{0}, q_{x}, y\right) \exp \left[-i\left(\omega_{0} t-q_{x} x\right)\right]$, which leads to

$$
R_{\alpha \beta}^{(1)}(\omega)=\frac{i\left(f_{\beta}-f_{\alpha}\right)<\alpha\left|V\left(\omega_{0}, q_{x}, y\right) e^{i q_{x} x}\right| \beta>}{\left(\omega-\omega_{0}\right)\left[E_{\beta}-E_{\alpha}+\hbar \omega+i \hbar / \tau\right]} .
$$

Taking the trace of $\hat{\rho}$ with the electron charge density operator, $e \delta(\mathbf{r}-\hat{\mathbf{r}})$, gives the wave charge density as

$$
\delta \rho(t, x, y) \equiv \rho(t, x, y)=\frac{e}{2 \pi} \int_{-\infty+i \eta}^{\infty+i \eta} d \omega e^{-i \omega t} \sum_{\alpha \beta} R_{\alpha \beta}^{(1)}(\omega) \psi_{\beta}^{*}(\mathbf{r}) \psi_{\alpha}(\mathbf{r}),
$$


where $\eta>0$. From Eqs. (6) and (7) it follows that $\rho(t, x, y)=\rho\left(t, q_{x}, y\right) \exp \left(i q_{x} x\right)$. Moreover, for $t / \tau \gg 1$, the contributions related to transitional processes are already negligible. It follows that $\rho\left(t, q_{x}, y\right)=\rho\left(\omega_{0}, q_{x}, y\right) \exp \left(-i \omega_{0} t\right)$.

Further, from Poisson's equation the wave electric potential $\phi\left(t, q_{x}, y\right)$ induced by $\rho\left(t, q_{x}, y\right)$ is given as

$$
\phi\left(t, q_{x}, y\right)=\frac{2}{\epsilon} \int_{-\infty}^{\infty} d y^{\prime} K_{0}\left(\left|q_{x} \| y-y^{\prime}\right|\right) \rho\left(t, q_{x}, y^{\prime}\right),
$$

where $K_{0}(x)$ is the modified Bessel function; $\phi$ and $\rho$ pertain to the $2 \mathrm{D}$ plane.

\section{Integral equation for EMP's}

For $t / \tau \gg 1$ the relation $\rho\left(t, q_{x}, y^{\prime}\right)=\rho\left(\omega_{0}, q_{x}, y^{\prime}\right) \exp \left(-i \omega_{0} t\right)$ holds; then from Eq. (8) it follows that $\phi\left(t, q_{x}, y\right)=$ $\phi\left(\omega_{0}, q_{x}, y\right) \exp \left(-i \omega_{0} t\right)$. In the absence of an external potential we have $V\left(\omega_{0}, q_{x}, y\right)=e \phi\left(\omega_{0}, q_{x}, y\right)$. As a result, from Eqs. (6)-(8), for $t / \tau \gg 1$, we obtain the integral equation for $\rho\left(\omega, q_{x}, y\right)$ as

$$
\begin{aligned}
& \rho\left(\omega, q_{x}, y\right)=\frac{2 e^{2}}{\epsilon L} \sum_{n_{\alpha}, n_{\beta}=0}^{\infty} \sum_{k_{x \alpha}} \frac{f_{\beta}-f_{\alpha}}{E_{\beta}-E_{\alpha}+\hbar \omega+i \hbar / \tau} \Pi_{n_{\alpha} n_{\beta}}\left(y, k_{x \alpha}, k_{x \beta}\right) \\
& \times \int_{-\infty}^{\infty} d \tilde{y} \int_{-\infty}^{\infty} d y^{\prime} \Pi_{n_{\alpha} n_{\beta}}\left(\tilde{y}, k_{x \alpha}, k_{x \beta}\right) K_{0}\left(\left|q_{x}\right|\left|\tilde{y}-y^{\prime}\right|\right) \rho\left(\omega, q_{x}, y^{\prime}\right),
\end{aligned}
$$

where $\Pi_{n_{\alpha} n_{\beta}}\left(y, k_{x \alpha}, k_{x \beta}\right)=\Psi_{n_{\alpha}}\left(y-y_{0}\left(k_{x \alpha}\right)\right) \Psi_{n_{\beta}}\left(y-y_{0}\left(k_{x \beta}\right)\right), k_{x \beta}=k_{x \alpha}-q_{x}$. We dropped the subscript 0 from $\omega_{0}$.

For flat LL's, i.e., for $\Omega \rightarrow 0$ and fixed width of the 2DEG, we first apply the Fourier transformation along $y$ to Eq. (9) and then carry out the sum over $k_{x \alpha}$ as well as the integral over $y^{\prime}$. This leads to $\rho\left(\omega, q_{x}, q_{y}\right) \epsilon_{l}\left(\omega, q_{x}, q_{y}\right) / \epsilon=0$, where $\epsilon_{l}\left(\omega, q_{x}, q_{y}\right)=\epsilon_{l}(\omega, \mathbf{q}) \equiv \epsilon_{l}(\omega, q)$ is the RPA longitudinal dielectric function $\left(\mathbf{q}=\left\{q_{x}, q_{y}\right\}\right)$. That is, for $\rho\left(\omega, q_{x}, q_{y}\right) \neq 0$ we have

$$
\frac{\epsilon_{l}(\omega, \mathbf{q})}{\epsilon}=1-\frac{2 \pi e^{2}}{\epsilon q L^{2}} \sum_{\alpha, \beta} \frac{f_{\beta}-f_{\alpha}}{E_{\beta}-E_{\alpha}+\hbar \omega+i \hbar / \tau}|<\alpha| e^{i \vec{q} \cdot \vec{r}}|\beta>|^{2}=0 .
$$

This equation gives the RPA dispersion relation for "bulk" longitudinal wave excitations of a 2DEG in a strong magnetic field. It also shows that the "bulk" excitations should have $\omega>\omega_{c}$. For definitness, we take $\omega>0$.

The integral equation (9) can be considered as a generalization of Eq. (10) of Ref. [11] since it takes into account nonlocal contributions to the current density $\propto \int d y^{\prime} \sigma_{\mu \gamma}\left(y, y^{\prime}\right) E_{\gamma}\left(y^{\prime}\right)$ and the screening by the edge and bulk states of the 2DEG. As shown in Ref. [15], the screening by edge states can be strong. We will obtain only solutions of Eq. (9) that are low in frequency, i.e., solutions for which $\omega / \omega_{c} \ll 1$. The appearence of any wave branch with $\omega<\omega_{c}$ is principally related to the existence of the edge of the 2DEG. Hence, any wave with $\omega<\omega_{c}$ should be localized at the edge and can be called edge magnetoplasmon.

We consider very low temperatures $T$ satisfying $\hbar v_{g n} \gg \ell_{0} k_{B} T$. Further, we will assume the long-wavelength limit $q_{x} \ell_{0} \ll 1$, which is well satisfied, e.g., for fundamental EMP [11] in the low-frequency region. Then if we compare the terms $\propto f_{\beta^{*}}$, for given $n_{\beta^{*}}$, on the RHS of Eq. (9), we obtain that the contribution to the sum over $n_{\alpha}$ with $n_{\alpha}=n_{\beta^{*}}$ is much larger than any other term of this sum or the sum of all terms with $n_{\alpha} \neq n_{\beta^{*}}$. The small parameter is $\left|\omega-q_{x} v_{g n_{\beta^{*}}}\left(k_{x \beta}\right)\right| / \omega_{c} \ll 1$, where $v_{g n_{\beta^{*}}}\left(k_{x \beta}\right)=\hbar^{-1} \partial E_{n_{\beta^{*}}}\left(k_{x \beta}\right) / \partial k_{x \beta}$ is the group velocity of any occupied state $\left\{n_{\beta^{*}}, k_{x \beta}\right\}$ of the $n_{\beta^{*}}$ LL. We assume that each occupied n-th LL has one intersection with the Fermi level at the edge of the channel and denote the group velocity of its edge states as $v_{g n} \equiv v_{g n}\left(k_{r n}\right)$. The small parameter given above implies $q_{x} v_{g 0} / \omega_{c} \ll 1$, as $v_{g 0}$ is typically the largest among $v_{g n}$. Similar results follow from an analysis of the terms $\propto f_{\alpha^{*}}$ in the sum over $n_{\beta}$ on the RHS of Eq. (9). Hence, for $\omega \ll \omega_{c}$ and $q_{x} v_{g 0} \ll \omega_{c}$ the terms with $n_{\alpha} \neq n_{\beta}$ can be neglected. This leads to the integral equation

$$
\begin{aligned}
& \rho\left(\omega, q_{x}, y\right)=\frac{2 e^{2}}{\epsilon L} \sum_{n_{\alpha}=0}^{\bar{n}} \sum_{k_{x \alpha}} \frac{f_{n_{\alpha}, k_{x \alpha}-q_{x}}-f_{n_{\alpha}, k_{x \alpha}}}{E_{n_{\alpha}, k_{x \alpha}-q_{x}}-E_{n_{\alpha}, k_{x \alpha}}+\hbar \omega+i \hbar / \tau} \Pi_{n_{\alpha} n_{\alpha}}\left(y, k_{x \alpha}, k_{x \alpha}-q_{x}\right) \\
& \times \int_{-\infty}^{\infty} d \tilde{y} \int_{-\infty}^{\infty} d y^{\prime} \Pi_{n_{\alpha} n_{\alpha}}\left(\tilde{y}, k_{x \alpha}, k_{x \alpha}-q_{x}\right) K_{0}\left(\left|q_{x}\right|\left|\tilde{y}-y^{\prime}\right|\right) \rho\left(\omega, q_{x}, y^{\prime}\right),
\end{aligned}
$$

where $\bar{n}$ denotes the highest occupied LL. For even $\nu$ the RHS of Eq. (11) should be multiplied by 2, the spin degeneracy factor. We now study EMPs following from Eq. (11). 


\section{EMPS FOR $\nu=1(2)$}

We first consider the case $\nu=1$ and then explain how the pertinent formulas should be modified for $\nu=2$. For $\nu=1$ we have $\bar{n}=0$ and Eq. (11) takes the form

$$
\begin{aligned}
& \rho\left(\omega, q_{x}, y\right)=\frac{e^{2}}{\pi \hbar \epsilon} \int_{-\infty}^{\infty} d k_{x \alpha} \frac{f_{0, k_{x \alpha}-q_{x}}-f_{0, k_{x \alpha}}}{\tilde{\omega}-v_{g 0}\left(k_{x \alpha}\right) q_{x}} \Pi_{00}\left(y, k_{x \alpha}, k_{x \alpha}-q_{x}\right) \\
& \times \int_{-\infty}^{\infty} d \tilde{y} \int_{-\infty}^{\infty} d y^{\prime} \Pi_{00}\left(\tilde{y}, k_{x \alpha}, k_{x \alpha}-q_{x}\right) K_{0}\left(\left|q_{x} \| \tilde{y}-y^{\prime}\right|\right) \rho\left(\omega, q_{x}, y^{\prime}\right),
\end{aligned}
$$

where $\tilde{\omega}=\omega+i / \tau$. As in Ref. [11, we seek a solution of Eq. (12) in the form

$$
\rho_{0}\left(\omega, q_{x}, y\right)=\Psi_{0}^{2}(\bar{y}) \sum_{n=0}^{\infty} \rho_{0}^{(n)}\left(\omega, q_{x}\right) H_{n}\left(\bar{y} / \ell_{0}\right)=\sum_{n=0}^{\infty} \sqrt{2^{n} n !} \rho_{0}^{(n)}\left(\omega, q_{x}\right) \Psi_{n}(\bar{y}) \Psi_{0}(\bar{y}) .
$$

Here $\bar{y}=y-y_{r 0}=y-y_{0}\left(k_{r 0}\right)$ and Eq. (13) is the exact solution due to the orthogonality of the Hermite polynomials $H_{n}(x)$. The expansion (13) is specific to $\nu=1(2)$ under the assumed conditions. This implies that the charge distortion far from the $\mathrm{LL}$ edge, $\gtrsim 10 \ell_{0}$, is much smaller than near this LL edge, $\lesssim \ell_{0}$. Physically this assumption can be well justified. We call the terms $n=0,1,2, \ldots$, the monopole, dipole, quadrupole, etc. terms in the expansion of $\rho_{0}\left(\omega, q_{x}, y\right)$ relative to $y=y_{r 0}$. Notice that expansion (13) can also be understood as an expansion in a complete set of oscillatory wave functions corresponding to all, occupied $(n=0)$ and empty $(n \geq 1)$ LLs.

We now multiply Eq. (12) by $H_{m}\left(\bar{y} / \ell_{0}\right)$ and integrate over $y$ from $y_{r 0}-\Delta y_{0}$ to $y_{r 0}+\Delta y_{0}$, where $\Delta y_{0} \gtrsim 3 \ell_{0}$. With the abbreviation $\rho_{0}^{(m)}\left(\omega, q_{x}\right) \equiv \rho_{0}^{(m)}$, we obtain

$$
\rho_{0}^{(m)}=\frac{e^{2}}{\pi \hbar \epsilon} \int_{-\infty}^{\infty} d k_{x \alpha} \frac{f_{0, k_{x \alpha}-q_{x}}-f_{0, k_{x \alpha}}}{\tilde{\omega}-v_{g 0}\left(k_{x \alpha}\right) q_{x}} d_{m 0}\left(q_{x}, \delta k_{x \alpha}\right) \sum_{n=0}^{\infty}\left(\frac{2^{n} n !}{2^{m} m !}\right)^{1 / 2} b_{n 0}^{(0)}\left(q_{x},\left|q_{x}\right|, \delta k_{x \alpha}\right) \rho_{0}^{(n)}
$$

where $\delta k_{x \alpha}=k_{x \alpha}-k_{r 0}, \delta k_{x \alpha^{\prime}}=\delta k_{x \alpha}-q_{x}$

$$
d_{m 0}\left(q_{x}, \delta k_{x \alpha}\right)=\frac{1}{\sqrt{2^{m} m !}} \int_{-\infty}^{\infty} d \bar{y} H_{m}\left(\bar{y} / \ell_{0}\right) \Pi_{00}\left(\bar{y}, \delta k_{x \alpha}, \delta k_{x \alpha^{\prime}}\right)
$$

and

$$
b_{n 0}^{(0)}\left(q_{x},\left|q_{x}\right|, \delta k_{x \alpha}\right)=\int_{-\infty}^{\infty} d \bar{y} \int_{-\infty}^{\infty} d \bar{y}^{\prime} \Pi_{00}\left(\bar{y}, \delta k_{x \alpha}, \delta k_{x \alpha^{\prime}}\right) K_{0}\left(\left|q_{x}\right|\left|\bar{y}-\bar{y}^{\prime}\right|\right) \Psi_{n}\left(\bar{y}^{\prime}\right) \Psi_{0}\left(\bar{y}^{\prime}\right)
$$

In both $d_{m 0}\left(q_{x}, \delta k_{x \alpha}\right)$ and $b_{n 0}^{(0)}\left(q_{x},\left|q_{x}\right|, \delta k_{x \alpha}\right)$ the first argument represents the $q_{x}$-dependent term of the argument of the wave function $\Psi_{0}\left(\bar{y}-\delta k_{x \alpha^{\prime}}\right)$ and, if replaced by 0 , it means this term can be neglected. We will also use the coefficients $a_{m n}\left(q_{x}\right)$ given by [1]

$$
a_{m n}\left(q_{x}\right)=a_{n m}\left(q_{x}\right)=\int_{-\infty}^{\infty} d x \Psi_{m}(x) \Psi_{0}(x) \int_{-\infty}^{\infty} d x^{\prime} K_{0}\left(\left|q_{x}\right|\left|x-x^{\prime}\right|\right) \Psi_{n}\left(x^{\prime}\right) \Psi_{0}\left(x^{\prime}\right),
$$

and satisfying $b_{n 0}^{(0)}\left(0,\left|q_{x}\right|, 0\right)=a_{n 0}\left(q_{x}\right)$

\section{A. Fundamental EMP}

Let us approximate the numerator on the RHS of Eq. (14) by the first nonzero term in the expansion over $q_{x}$ : $f_{0, k_{x \alpha}-q_{x}}-f_{0, k_{x \alpha}} \approx-q_{x}\left(\partial f_{0, k_{x \alpha}} / \partial k_{x \alpha}\right)=\delta\left(k_{x \alpha}-k_{r 0}\right) q_{x}$. Then Eq. (14), after integration over $k_{x \alpha}$, gives

$$
\rho_{0}^{(m)}=\frac{e^{2}}{\pi \hbar \epsilon} \frac{q_{x}}{\tilde{\omega}_{0}} d_{m 0}\left(q_{x}, 0\right) \sum_{n=0}^{\infty}\left(\frac{2^{n} n !}{2^{m} m !}\right)^{1 / 2} b_{n 0}^{(0)}\left(q_{x},\left|q_{x}\right|, 0\right) \rho_{0}^{(n)}
$$


where $\tilde{\omega}_{0}=\left(\tilde{\omega}-v_{g 0} q_{x}\right)$. Further, if we neglect a small term $\propto q_{x} \ell_{0}$ in the argument of the wave function $\Psi_{0}\left(\bar{y}+\ell_{0}^{2} q_{x}\right)$, we obtain $d_{m 0}\left(q_{x}, 0\right) \approx d_{m 0}(0,0)=\delta_{m, 0}$ and $b_{n 0}^{(0)}\left(q_{x},\left|q_{x}\right|, 0\right) \approx b_{n 0}^{(0)}\left(0,\left|q_{x}\right|, 0\right)=a_{n 0}\left(q_{x}\right)$. Substituting the former in Eq. (18), we obtain $\rho_{0}^{(m)} \equiv 0$ for $m \geq 1$; thus, only $\rho_{0}^{(0)}$ can be finite. Then Eq. (18) can be rewritten as

$$
\rho_{0}^{(0)}=\frac{e^{2}}{\pi \hbar \epsilon} \frac{q_{x}}{\tilde{\omega}_{0}} a_{00}\left(q_{x}\right) \rho_{0}^{(0)} .
$$

For $\rho_{0}^{(0)} \neq 0$ Eq. (19) gives the dispersion relation

$$
\omega=q_{x} v_{g 0}+\frac{2}{\epsilon} \sigma_{y x}^{0} q_{x}\left[\ln \left(\frac{1}{\left|q_{x}\right| \ell_{0}}\right)+\frac{3}{4}\right]-\frac{i}{\tau},
$$

where $\sigma_{y x}^{0}=e^{2} / 2 \pi \hbar$ for $\nu=1$ and $\sigma_{y x}^{0}=e^{2} / \pi \hbar$ for $\nu=2$. We have used the result $a_{00}\left(q_{x}\right) \approx\left[\ln \left(1 /\left|q_{x}\right| \ell_{0}\right)+3 / 4\right]$ [1] for $\left|q_{x}\right| \ell_{0} \ll 1$. If we neglect the dissipation, the dispersion relation (20) and the corresponding charge density profile coincide with the corresponding expressions for the fundamental mode obtained in Ref. [11].

Untill now nonlocal effects and screening by the 2DEG have not changed the results for the fundamental EMP of Ref. 111. In Ref. 11 it is shown that the next term that can affect the fundamental mode of the $n=0$ LL is the quadrupole term. Then, if we neglect dissipation $\omega-q_{x} v_{g 0}$ becomes slightly larger, by a factor $\left[1+0.125 / a_{00}^{2}\left(q_{x}\right)\right] \approx 1$. Typically we have $a_{00}\left(q_{x}\right) \gtrsim 10$. In addition, the spatial structure of the fundamental EMP acquires a small quadrupole term $\left|\rho_{0}^{(2)} / \rho_{0}^{(0)}\right| \approx 1 / 8 a_{00}\left(q_{x}\right) \ll 1$ [11]. It can be shown from Eqs. (14)-(16) that here too the corrections to the fundamental EMP due to the quadrupole term are very small; namely, $a_{00}\left(q_{x}\right)$ on the RHS of Eq. (200) should be changed to $a_{00}\left(q_{x}\right)+a_{20}\left(q_{x}\right) q_{x}^{2} \ell_{0}^{2} / 2 \sqrt{2} \approx a_{00}\left(q_{x}\right)-q_{x}^{2} \ell_{0}^{2} / 8$. Further, the spatial structure of the fundamental EMP acquires a small quadrupole term: $\rho_{0}^{(2)} / \rho_{0}^{(0)}=q_{x}^{2} \ell_{0}^{2} / 8 \ll 1$. Thus, for weak dissipation the results of Ref. [1] for the fundamental EMP are nearly the same as those of the present microscopic treatment.

\section{B. Dipole EMP}

For a more acurate calculation of the RHS of Eq. (14) we must take into account higher order terms in the expansion over $q_{x}$. As shown below, when this is done it leads to additional branches. To obtain the dipole branch correctly we must keep the first three nonzero terms in the expansion of the numerator of RHS of Eq. (14) over $q_{x}$ by writing

$$
f_{0, k_{x \alpha}-q_{x}}-f_{0, k_{x \alpha}} \approx\left[q_{x}-\frac{q_{x}^{2}}{2 !} \frac{\partial}{\partial k_{x \alpha}}+\frac{q_{x}^{3}}{3 !} \frac{\partial^{2}}{\partial k_{x \alpha}^{2}}\right] \delta\left(k_{x \alpha}-k_{r 0}\right) .
$$

Further, we will consider only the first two terms, $n=0$ and $n=1$ in the sum of Eq. (13). Then from Eq. (14) for $m=0$ and $m=1$ we obtain the following system of linear equations for $\rho_{0}^{(0)}$ and $\rho_{0}^{(1)}$

$$
\begin{aligned}
& \rho_{0}^{(0)}=\frac{e^{2}}{\pi \hbar \epsilon \tilde{\omega}_{0}} \int_{-\infty}^{\infty} d k_{x \alpha}\left(f_{0, k_{x \alpha}-q_{x}}-f_{0, k_{x \alpha}}\right) d_{00}\left(q_{x}, \delta k_{x \alpha}\right) \sum_{n=0}^{1}\left(2^{n} n !\right)^{1 / 2} b_{n 0}^{(0)}\left(q_{x},\left|q_{x}\right|, \delta k_{x \alpha}\right) \rho_{0}^{(n)}, \\
& \rho_{0}^{(1)}=\frac{e^{2}}{\pi \hbar \epsilon \tilde{\omega}_{0}} \int_{-\infty}^{\infty} d k_{x \alpha}\left(f_{0, k_{x \alpha}-q_{x}}-f_{0, k_{x \alpha}}\right) d_{10}\left(q_{x}, \delta k_{x \alpha}\right) \sum_{n=0}^{1}\left(\frac{2^{n} n !}{2^{1} 1 !}\right)^{1 / 2} b_{n 0}^{(0)}\left(q_{x},\left|q_{x}\right|, \delta k_{x \alpha}\right) \rho_{0}^{(n)} .
\end{aligned}
$$

We assumed that, for $T \rightarrow 0, v_{g 0}\left(k_{x \alpha}\right)$ is independent of $k_{x \alpha}$ in the vicinity of $k_{x \alpha}=k_{r 0}$ and replaced it by $v_{g 0}$. This assumption does not impose any important restriction on the analysis. Therefore, taking into account Eq. (21) we can replace $1 /\left(\tilde{\omega}-v_{g 0}\left(k_{x \alpha}\right) q_{x}\right)$ by $1 /\left(\tilde{\omega}-q_{x} v_{g 0}\right)$ and place it in front of the integrals in Eqs. 222)-(23).

In Eq. (22) we can assume $d_{00}\left(q_{x}, \delta k_{x \alpha}\right)=1$ since corrections to it, varying as $\sim\left(q_{x} \ell_{0}\right)^{2}$, are not essential. To evaluate the contribution $\propto \rho_{0}^{(0)}$ on the RHS of Eq. (22) it is sufficient to keep only the first term, $\propto q_{x}$, on the RHS of Eq. (21). This gives

$$
\rho_{0}^{(0)}=\frac{e^{2}}{\pi \hbar \epsilon \tilde{\omega}_{0}}\left\{q_{x} b_{00}^{(0)}\left(0,\left|q_{x}\right|, 0\right) \rho_{0}^{(0)}+\sqrt{2} I_{01}^{(1)}\left(q_{x}\right) \rho_{0}^{(1)}\right\},
$$

where 


$$
I_{01}^{(1)}\left(q_{x}\right)=\int_{-\infty}^{\infty} d k_{x \alpha}\left(f_{0, k_{x \alpha}-q_{x}}-f_{0, k_{x \alpha}}\right) b_{10}^{(0)}\left(q_{x},\left|q_{x}\right|, \delta k_{x \alpha}\right)
$$

and where $b_{00}^{(0)}\left(q_{x},\left|q_{x}\right|, 0\right)$ has justifiably been approximated by $b_{00}^{(0)}\left(0,\left|q_{x}\right|, 0\right)$. Before calculating $I_{01}^{(1)}\left(q_{x}\right)$ we notice that the approximation for $d_{00}\left(q_{x}, \delta k_{x \alpha}\right)$ entails the neglect of small corrections to $I_{01}^{(1)}\left(q_{x}\right)$ proportional to $\left(q_{x} \ell_{0}\right)^{k}$ if $k \geq 4$. Then Eqs. (21) and Eq. (25) give

$$
\begin{aligned}
I_{01}^{(1)}\left(q_{x}\right)= & -\frac{1}{\sqrt{2} \ell_{0}}\left(q_{x} \ell_{0}\right)^{2} a_{11}\left(q_{x}\right)+ \\
& +\frac{q_{x}^{2}}{2} \int_{-\infty}^{\infty} d k_{x \alpha} \delta\left(k_{x \alpha}-k_{r 0}\right)\left[1+\frac{q_{x}}{3} \frac{\partial}{\partial k_{x \alpha}}\right] \frac{\partial}{\partial k_{x \alpha}} b_{10}^{(0)}\left(q_{x},\left|q_{x}\right|, \delta k_{x \alpha}\right) .
\end{aligned}
$$

The corrections $\propto\left(q_{x} \ell_{0}\right)^{k}, k \geq 4$, we neglected in the first term on the RHS are related to the expansion

$$
\Psi_{0}\left(\bar{y}+\ell_{0}^{2} q_{x}\right)=\Psi_{0}(\bar{y})-\frac{1}{\sqrt{2}} \Psi_{1}(\bar{y})\left(q_{x} \ell_{0}\right)+\frac{1}{4}\left[\sqrt{2} \Psi_{2}(\bar{y})-\Psi_{0}(\bar{y})\right]\left(q_{x} \ell_{0}\right)^{2}+\ldots
$$

It can be shown that the term $\left(q_{x} / 3\right) \partial / \partial k_{x \alpha}$ in the square brackets [... of Eq. (26) gives, upon integration over $k_{x \alpha}$, a vanishing contribution since the remaining double integral changes sign upon making the changes $\bar{y} \rightarrow-\bar{y}$ and $\bar{y}^{\prime} \rightarrow-\bar{y}^{\prime}$. As for the term corresponding to 1 in [...], its evaluation gives $q_{x}^{2} \ell_{0} a_{11}\left(q_{x}\right) / \sqrt{2}$. Hence, the two finite terms on the RHS of Eq. (26) cancel each other. The final result is $I_{01}^{(1)}\left(q_{x}\right)=0$ and Eq. (24) takes the form of Eq. (19). That is, in this approximation the fundamental mode is purely monopole and is totally independent of dipole excitations.

We now consider Eq. (23). Here we should consider six different contributions $I_{1 n}^{(1)}\left(q_{x}\right), I_{1 n}^{(2)}\left(q_{x}\right)$, and $I_{1 n}^{(3)}\left(q_{x}\right)$ for $n=0,1$ related, respectively, to the first $\left(\propto q_{x}\right)$, second $\left(\propto q_{x}^{2}\right)$, and third $\left(\propto q_{x}^{3}\right)$ term on the RHS of Eq. (21). Then Eq. (23) can be rewritten as

$$
\rho_{0}^{(1)}=\frac{e^{2}}{\pi \hbar \epsilon \tilde{\omega}_{0}} \sum_{n=0}^{1} \sum_{l=1}^{3} I_{1 n}^{(l)}\left(q_{x}\right) \rho_{0}^{(n)}
$$

where

$$
\begin{gathered}
I_{1 n}^{(1)}\left(q_{x}\right)=\left(\frac{2^{n} n !}{2^{1} 1 !}\right)^{1 / 2} q_{x} d_{10}\left(q_{x}, 0\right) b_{n 0}^{(0)}\left(q_{x},\left|q_{x}\right|, 0\right), \\
I_{1 n}^{(2)}\left(q_{x}\right)=-\frac{\sqrt{2^{n-1} n !}}{2 !} q_{x}^{2} \int_{-\infty}^{\infty} d k_{x \alpha} d_{10}\left(q_{x}, \delta k_{x \alpha}\right) b_{n 0}^{(0)}\left(q_{x},\left|q_{x}\right|, \delta k_{x \alpha}\right) \frac{\partial}{\partial k_{x \alpha}} \delta\left(k_{x \alpha}-k_{r 0}\right), \\
I_{1 n}^{(3)}\left(q_{x}\right)=\frac{\sqrt{2^{n-1} n !}}{3 !} q_{x}^{3} \int_{-\infty}^{\infty} d k_{x \alpha} d_{10}\left(q_{x}, \delta k_{x \alpha}\right) b_{n 0}^{(0)}\left(q_{x},\left|q_{x}\right|, \delta k_{x \alpha}\right) \frac{\partial^{2}}{\partial k_{x \alpha}^{2}} \delta\left(k_{x \alpha}-k_{r 0}\right) .
\end{gathered}
$$

Again we will neglect contributions $\propto\left(q_{x} \ell_{0}\right)^{m}$ for $m \geq 4$. In Eq. (29) we have $d_{10}\left(q_{x}, 0\right)=-\left(q_{x} \ell_{0}\right) / \sqrt{2}+O\left(\left(q_{x} \ell_{0}\right)^{3}\right)$ and $b_{00}^{(0)}\left(q_{x},\left|q_{x}\right|, 0\right)=a_{00}\left(q_{x}\right)+O\left(\left(q_{x} \ell_{0}\right)^{2}\right), b_{10}^{(0)}\left(q_{x},\left|q_{x}\right|, 0\right)=-\left(q_{x} \ell_{0} / \sqrt{2}\right) a_{11}\left(q_{x}\right)+O\left(\left(q_{x} \ell_{0}\right)^{3}\right)$. It follows

$$
I_{10}^{(1)}\left(q_{x}\right)=-\frac{\left(q_{x} \ell_{0}\right)^{2}}{2 \ell_{0}} a_{00}\left(q_{x}\right), \quad I_{11}^{(1)}\left(q_{x}\right)=\frac{\left(q_{x} \ell_{0}\right)^{3}}{2 \ell_{0}} a_{11}\left(q_{x}\right) .
$$

Evaluating the integral in Eq. (30) by parts we obtain

$$
\begin{aligned}
I_{1 n}^{(2)}\left(q_{x}\right)= & \frac{\left(2^{n-1} n !\right)^{1 / 2}}{2 !} q_{x}^{2}\left\{\frac{d}{d \delta k_{x \alpha}}\left[d_{10}\left(q_{x}, \delta k_{x \alpha}\right)\right]_{\mid \delta k_{x \alpha}=0} b_{n 0}^{(0)}\left(q_{x},\left|q_{x}\right|, 0\right)+\right. \\
& \left.d_{10}\left(q_{x}, 0\right) \frac{d}{d \delta k_{x \alpha}}\left[b_{n 0}^{(0)}\left(q_{x},\left|q_{x}\right|, \delta k_{x \alpha}\right)\right]_{\mid \delta k_{x \alpha}=0}\right\} .
\end{aligned}
$$

This gives the contributions 


$$
I_{10}^{(2)}\left(q_{x}\right)=\frac{\left(q_{x} \ell_{0}\right)^{2}}{2 \ell_{0}} a_{00}\left(q_{x}\right), \quad I_{11}^{(2)}\left(q_{x}\right)=-\frac{\left(q_{x} \ell_{0}\right)^{3}}{\ell_{0}} a_{11}\left(q_{x}\right) .
$$

Evaluating the integral in Eq. (31) by parts we obtain

$$
I_{10}^{(3)}\left(q_{x}\right)=0, \quad I_{11}^{(3)}\left(q_{x}\right)=\frac{2\left(q_{x} \ell_{0}\right)^{3}}{3 \ell_{0}} a_{11}\left(q_{x}\right) .
$$

Using Eqs. (32), (34) and (35) we can rewrite Eq. (28) as

$$
\rho_{0}^{(1)}=\frac{e^{2} \ell_{0}^{2} a_{11}\left(q_{x}\right)}{6 \pi \hbar \epsilon \tilde{\omega}_{0}} q_{x}^{3} \rho_{0}^{(1)} .
$$

Eq. (36) shows that the dipole branch, similar to that in Ref. [11, is not coupled to monopole excitations of the charge density. Further, Eq. (36), with $a_{11}\left(q_{x}\right)=1 / 2$ for $\left|q_{x}\right| \ell_{0} \ll 1$ and precision $\lesssim 0.2 \%$ [1], gives the dispersion relation of the dipole branch, $\rho_{0}^{(1)}\left(\omega, q_{x}\right) \neq 0$,

$$
\omega=v_{g 0} q_{x}+\frac{\ell_{0}^{2}}{6 \epsilon} \sigma_{y x}^{0} q_{x}^{3}-\frac{i}{\tau}
$$

where $\sigma_{y x}^{0}=e^{2} / 2 \pi \hbar$ for $\nu=1$ and $\sigma_{u x}^{0}=e^{2} / \pi \hbar$ for $\nu=2$.

An important difference of Eq. (37) from the corresponding result, Eq. (40), of Ref. 11] for the pure dipole EMP is that here we have $\omega-v_{g 0} q_{x} \propto q_{x}^{3}$ whereas in Ref. 11] this becomes $\omega-v_{g 0} q_{x} \propto q_{x}$. That is, the term caused by the Coulomb interaction has a nonacoustic behavior if we neglect dissipation. The calculations demonstrate clearly that the spatial dispersion effects, although they depend on $q_{x}$, they are essentially related to the wave structure along the $y$-direction. It is known that spatial dispersion is directly related to the nonlocality of responses, as expressed by the dielectric function, etc. [16]. In Eq. (37) both nonlocal effects and edge states screening are taken into account. We point out that in Ref. 17] a similar dispersion law, $\omega \propto a q_{x}^{3}$, was obtained for short-range forces, i.e., for an interaction that is essentially not of the Coulomb type.

\section{FUNDAMENTAL EMPS FOR $\nu=4$ AND 6}

For $\nu=2(\bar{n}+1)$ the $n=0, \ldots, n=\bar{n}$ LLs have intersections with the Fermi level at $y_{r 0}, \ldots, y_{r \bar{n}}$, respectively, and Eq. (11) can be written as

$$
\begin{aligned}
\rho\left(\omega, q_{x}, y\right) & =\frac{2 e^{2}}{\pi \hbar \epsilon} \sum_{n=0}^{\bar{n}} \int_{-\infty}^{\infty} d k_{x \alpha} \frac{f_{n, k_{x \alpha}-q_{x}}-f_{n, k_{x \alpha}}}{\tilde{\omega}-v_{g n}\left(k_{x \alpha}\right) q_{x}} \Pi_{n n}\left(y, k_{x \alpha}, k_{x \alpha}-q_{x}\right) \\
& \left.\times \int_{-\infty}^{\infty} d \tilde{y} \int_{-\infty}^{\infty} d y^{\prime} \Pi_{n n}\left(\tilde{y}, k_{x \alpha}, k_{x \alpha}-q_{x}\right) K_{0}\left(\left|q_{x}\right|\left|\tilde{y}-y^{\prime}\right|\right)\right] \rho\left(\omega, q_{x}, y^{\prime}\right) .
\end{aligned}
$$

For confining potentials smooth on the $\ell_{0}$ scale we have $\Delta y_{m-1, m}=y_{r m-1}-y_{r m} \gg \ell_{0}$, where $m \leq \bar{n}$. For $\nu=4$ there is only one inter-LL length $\Delta y_{0,1}=y_{r 0}-y_{r 1}$. Further, making the same approximations, in the long-wavelength limit $q_{x} \ell_{0} \ll 1$, as in Sec. IIIA and integrating over $k_{x \alpha}$ in Eq. (38), we obtain

$$
\begin{aligned}
\rho\left(\omega, q_{x}, y\right)= & \frac{2 e^{2}}{\pi \hbar \epsilon} \sum_{n=0}^{\bar{n}} \frac{q_{x}}{\tilde{\omega}_{n}} \Psi_{n}^{2}\left(y-y_{r n}\right) \\
& \times \int_{-\infty}^{\infty} d \tilde{y} \int_{-\infty}^{\infty} d y^{\prime}\left[\Psi_{n}^{2}\left(\tilde{y}-y_{r n}\right) K_{0}\left(\left|q_{x}\right|\left|\tilde{y}-y^{\prime}\right|\right)\right] \rho\left(\omega, q_{x}, y^{\prime}\right),
\end{aligned}
$$

where $\tilde{\omega}_{n} \equiv \tilde{\omega}-v_{g n} q_{x}$. It follows that $\rho\left(\omega, q_{x}, y\right)$ can be represented by a sum of charges $\rho_{n}\left(\omega, q_{x}, y\right)=\rho_{n}^{(0)}\left(\omega, q_{x}\right) \Psi_{n}^{2}(y-$ $\left.y_{r n}\right)$, localized at the edge of the $n$-th LL, within a region of extent $\sim \sqrt{2 n+1} \ell_{0}, n=0, \ldots, \bar{n}$. The result is

$$
\rho\left(\omega, q_{x}, y\right)=\sum_{n=0}^{\bar{n}} \rho_{n}^{(0)}\left(\omega, q_{x}\right) \Psi_{n}^{2}\left(y-y_{r n}\right) .
$$

We substitute Eq. (40) into Eq. (39) and demand that the coefficients of $\Psi_{n}^{2}\left(y-y_{r n}\right)$ on both sides of Eq. (39) be equal. This leads to $\bar{n}+1$ linear homogeneous equations for $\rho_{n}^{(0)}\left(\omega, q_{x}\right)$. 


$$
\text { A. } \nu=4
$$

Due to $\Delta y_{0,1} \gg \ell_{0}$ we can neglect the exponentially small overlap between $\rho_{0}\left(\omega, q_{x}, y\right)$ and $\rho_{1}\left(\omega, q_{x}, y\right)$. We will assume $q_{x} \Delta y_{01} \ll 1$. As shown above, from Eqs. (39) and (40), with $\bar{n}=1$, we obtain the system

$$
\begin{gathered}
\rho_{0}^{(0)}=\frac{2 e^{2}}{\pi \hbar \epsilon} \frac{q_{x}}{\tilde{\omega}_{0}}\left[a_{00}\left(q_{x}\right) \rho_{0}^{(0)}+a_{00}^{11}\left(q_{x}, \Delta y_{01}\right) \rho_{1}^{(0)}\right], \\
\rho_{1}^{(0)}=\frac{2 e^{2}}{\pi \hbar \epsilon} \frac{q_{x}}{\tilde{\omega}_{1}}\left[a_{00}^{11}\left(q_{x}, \Delta y_{01}\right) \rho_{0}^{(0)}+a_{11}^{11}\left(q_{x}, 0\right) \rho_{1}^{(0)}\right],
\end{gathered}
$$

where $\rho_{n}^{(0)}\left(\omega, q_{x}\right) \equiv \rho_{n}^{(0)}$ and

$$
a_{n n}^{m m}\left(q_{x}, \Delta y\right)=\int_{-\infty}^{\infty} \int_{-\infty}^{\infty} d x d x^{\prime} \Psi_{n}^{2}(x) \Psi_{m}^{2}\left(x^{\prime}\right) K_{0}\left(\left|q_{x}\right|\left|x-x^{\prime}+\Delta y\right|\right) .
$$

Here $a_{n n}^{m m}\left(q_{x}, \Delta y\right)=a_{m m}^{n n}\left(q_{x}, \Delta y\right), a_{n n}^{m m}\left(q_{x}, \Delta y\right)=a_{n n}^{m m}\left(q_{x},-\Delta y\right)$ and $a_{00}^{00}\left(q_{x}, 0\right)=a_{00}\left(q_{x}\right)$. For a nontrivial solution of the system of Eqs. (41) and (42) the determinant of the coefficients must vanish. This gives the dispersion relation of the renormalized fundamental EMP of the $n=0 \mathrm{LL}$ as

$$
\omega=q_{x} v_{01}+\frac{\sigma_{y x}^{0}}{\epsilon} q_{x}\left[2 \ln \left(\frac{1}{\left|q_{x}\right| \ell_{0}}\right)-\ln \left(\frac{\Delta y_{01}}{\ell_{0}}\right)+\frac{3}{5}\right]-\frac{i}{\tau},
$$

and that of the $n=1 \mathrm{LL}$ as

$$
\omega=q_{x} v_{01}+\frac{\sigma_{y x}^{0}}{\epsilon} q_{x}\left[\ln \left(\frac{\Delta y_{01}}{\ell_{0}}\right)+\frac{2}{5}\right]-\frac{i}{\tau},
$$

where $\sigma_{y x}^{0}=2 e^{2} / \pi \hbar$ and $v_{01}=\left(v_{g 0}+v_{g 1}\right) / 2$. All double integrals involved in the coefficients of Eqs. (41) and (42) can be evaluated within the approximation $K_{0}(x) \approx \ln (2 /|x|)-\gamma$, where $\gamma$ is the Euler constant. We obtain $a_{11}^{\pi 1}\left(q_{x}, 0\right) \approx \ln \left(1 /\left|q_{x}\right| \ell_{0}\right)+1 / 4$ and $a_{00}^{11}\left(q_{x}, \Delta y_{01}\right) \approx(\ln (2)-\gamma)-\ln \left(\left|q_{x}\right| \Delta y_{01}\right) \approx \ln \left(1 /\left|q_{x} \Delta y_{01}\right|\right)+0.1$. Notice that in the absence of dissipation Eqs. (44) and (45) coincide with the quasi-microscopic results of Ref. [18]. ¿From Eqs. (41) and (42) it is easy to see that, if the inter-LL Coulomb coupling is neglected by setting $a_{00}^{11}\left(q_{x}, \Delta y_{01}\right) \equiv 0$, the dispersion laws of the decoupled fundamental EMPs of the $n=0$ and $n=1$ LLs are given, respectively, by Eq. (20) and by

$$
\omega=q_{x} v_{g 1}+\frac{2 e^{2}}{\pi \hbar \epsilon} q_{x}\left[\ln \left(\frac{1}{\left|q_{x}\right| \ell_{0}}\right)+\frac{1}{4}\right]-\frac{i}{\tau} .
$$

Substituting Eq. (44) in Eq. (41) and Eq. (45) in Eq. (42) we obtain, respectively, $\rho_{1}^{(0)} / \rho_{0}^{(0)} \approx 1$ and $\rho_{0}^{(0)} / \rho_{1}^{(0)} \approx-1$. This means that in the former case the wave charges localized at the edges of the $n=0$ LL and $n=1$ LLs are in phase whereas in the latter they are out of phase. Therefore, the former EMP has an acoustic spatial structure along the $y$ axis while the latter EMP has an optical spatial structure though its dispersion law is purely acoustic.

$$
\text { B. } \nu=6
$$

For $\nu=6$ we have the intersection of the $n=2 \mathrm{LL}$ with the Fermi level, at $y_{r 2}$, in addition those of the $n=0$ and $n=1 \mathrm{LLs} ; \bar{n}=2$. Corresponding to Eqs. (39) and Eq. (40) we now obtain

$$
\begin{gathered}
\rho_{0}^{(0)}=\frac{2 e^{2}}{\pi \hbar \epsilon} \frac{q_{x}}{\tilde{\omega}_{0}}\left[a_{00}\left(q_{x}\right) \rho_{0}^{(0)}+a_{00}^{11}\left(q_{x}, \Delta y_{01}\right) \rho_{1}^{(0)}+a_{00}^{22}\left(q_{x}, \Delta y_{02}\right) \rho_{2}^{(0)}\right], \\
\rho_{1}^{(0)}=\frac{2 e^{2}}{\pi \hbar \epsilon} \frac{q_{x}}{\tilde{\omega}_{1}}\left[a_{00}^{11}\left(q_{x}, \Delta y_{01}\right) \rho_{0}^{(0)}+a_{11}^{11}\left(q_{x}, 0\right) \rho_{1}^{(0)}+a_{11}^{22}\left(q_{x}, \Delta y_{12}\right) \rho_{2}^{(0)}\right], \\
\rho_{2}^{(0)}=\frac{2 e^{2}}{\pi \hbar \epsilon} \frac{q_{x}}{\tilde{\omega}_{2}}\left[a_{00}^{22}\left(q_{x}, \Delta y_{02}\right) \rho_{0}^{(0)}+a_{11}^{22}\left(q_{x}, \Delta y_{12}\right) \rho_{1}^{(0)}+a_{22}^{22}\left(q_{x}, 0\right) \rho_{2}^{(0)}\right] .
\end{gathered}
$$


The vanishing of the determinant of the coefficients leads to the cubic equation

$$
\omega^{3}+a_{2}\left(q_{x}\right) \omega^{\prime 2}+a_{1}\left(q_{x}\right) \omega^{‘}+a_{0}\left(q_{x}\right)=0,
$$

where $\omega^{6}=\tilde{\omega} /\left(2 e^{2} q_{x} / \pi \hbar \epsilon\right)$. The expressions for the coefficients $a_{k}\left(q_{x}\right), k=0,1,2$, are given in the appendix. We will assume that $q_{x} \Delta y_{i j} \ll 1, i \neq j \leq 2$.

It can be shown that all three roots of Eq. (50) are real and different as they correspond to the irreducible case. Assuming $\Delta y_{02}, \Delta y_{12}, \Delta y_{01} \gg \ell_{0}$, these roots give the dispersion law for the renormalized fundamental EMP of the $n=0 \mathrm{LL}$ as

$$
\omega=q_{x} v_{012}+\frac{2 \sigma_{y x}^{0}}{3 \epsilon} q_{x}\left\{3 \ln \left(\frac{1}{\left|q_{x}\right| \ell_{0}}\right)-\frac{2}{3}\left[\ln \left(\frac{\Delta y_{02}}{\ell_{0}}\right)+\ln \left(\frac{\Delta y_{01}}{\ell_{0}}\right)+\ln \left(\frac{\Delta y_{12}}{\ell_{0}}\right)\right]+\frac{8}{15}\right\}-\frac{i}{\tau},
$$

and that for the renormalized fundamental EMP of the $n=1 \mathrm{LL}, \omega_{+}$, and $n=2 \mathrm{LL}, \omega_{-}$, as

$$
\begin{aligned}
\omega_{ \pm}= & q_{x} v_{012}+\frac{2 \sigma_{y x}^{0}}{9 \epsilon} q_{x}\left\{\ln \left(\frac{\Delta y_{02}}{\ell_{0}}\right)+\ln \left(\frac{\Delta y_{01}}{\ell_{0}}\right)+\ln \left(\frac{\Delta y_{12}}{\ell_{0}}\right)+\frac{7}{10}\right\} \pm \\
& \frac{2 \sqrt{2} \sigma_{y x}^{0}}{9 \epsilon} q_{x}\left\{\ln ^{2}\left(\frac{\Delta y_{02}}{\Delta y_{01}}\right)+\ln ^{2}\left(\frac{\Delta y_{02}}{\Delta y_{12}}\right)+\ln ^{2}\left(\frac{\Delta y_{01}}{\Delta y_{12}}\right)+A\right\}^{1 / 2}-\frac{i}{\tau} .
\end{aligned}
$$

Here $\sigma_{y x}^{0}=3 e^{2} / \pi \hbar, v_{012}=\left(v_{g 0}+v_{g 1}+v_{g 2}\right) / 3$, and

$$
\begin{aligned}
A= & \left(\tilde{v}_{g 0}+3 / 4\right)\left[\left(\tilde{v}_{g 0}-\tilde{v}_{g 2}+3 / 4\right) / 2+\ln \left(\Delta y_{02} \Delta y_{01} / \Delta y_{12}{ }^{2}\right)\right] \\
& +\left(\tilde{v}_{g 1}+1 / 4\right)\left[\left(\tilde{v}_{g 1}-\tilde{v}_{g 0}-1 / 2\right) / 2+\ln \left(\Delta y_{01} \Delta y_{12} / \Delta y_{02}{ }^{2}\right)\right] \\
& +\tilde{v}_{g 2}\left[\left(\tilde{v}_{g 2}-\tilde{v}_{g 1}-1 / 4\right) / 2+\ln \left(\Delta y_{02} \Delta y_{12} / \Delta y_{01}{ }^{2}\right)\right]
\end{aligned}
$$

where $\tilde{v}_{g i}=v_{g i} /\left(2 e^{2} / \pi \hbar \epsilon\right), i=0,1,2$. Notice that the fundamental EMPs of the $n=1$ and $n=2$ LLs have purely acoustic dispersion laws, cf. Eq. (52). Only the fundamental EMP of the $n=0$ LL, Eq. (51), behaves in the previously obtained manner $\propto q_{x} \ln \left(q_{x}\right)$, cf. Refs. [1] - [10]), for a fundamental EMP. It is difficult to clearly disentangle the contributions of the $n=2 \mathrm{LL}$ in Eq. (52). It can be shown though that, if they are neglected by setting $\tilde{a}_{22}^{22}, a_{11}^{22}$, $a_{00}^{22}, v_{g 2}$ to zero, then we have $\omega_{2}^{\prime}=0$ and Re $\omega_{-}=0$ as we should in this limit.

The solutions of the cubic equation, Eqs. (51) and (52), are not obtained using the standard expressions, reproduced in the appendix, which become very unwieldy for the present case. Instead, the following reasoning is used. If $\omega$ has an acoustic character for some branches, then $\omega^{\prime}$ in Eq. (50) is independent of $q_{x}$ and should satisfy the quadratic equation obtained by differentiating Eq. (50) with respect to $q_{x}$

$$
\frac{\partial a_{2}\left(q_{x}\right)}{\partial q_{x}} \omega^{2}+\frac{\partial a_{1}\left(q_{x}\right)}{\partial q_{x}} \omega+\frac{\partial a_{0}\left(q_{x}\right)}{\partial q_{x}}=0 .
$$

The two roots $\omega_{2}^{\prime}$ and $\omega_{3}^{\prime}$ of Eq. (54) are indeed independent of $q_{x}$ and lead to the solutions $\omega_{-}$and $\omega_{+}$, respectively, given by Eq. (52). The other root is given by $\omega_{1}^{\prime}=-a_{2}\left(q_{x}\right)-\left(\omega_{2}^{\prime}+\omega_{3}^{\prime}\right)$ and results in Eq. (51). Of course both methods give the same solutions $\omega_{j}^{\prime}, j=1,2,3$. Notice that on the RHS of Eqs. (51) and (52) we used approximate expressions of $a_{i i}^{j j}\left(q_{x}, \Delta y_{i j}\right)$ which, assuming $\Delta y_{i j} / \ell_{0} \gg 1, i \neq j$, are obtained with the approximation

$$
\int_{-\infty}^{\infty} \int_{-\infty}^{\infty} d x d x^{\prime} \Psi_{i}^{2}(x) \Psi_{j}^{2}\left(x^{\prime}\right) \ln \left(\left|x-x^{\prime}+\Delta y_{i j}\right| / \ell_{0}\right) \approx \ln \left(\Delta y_{i j} / \ell_{0}\right) .
$$

Below we will demonstrate that the approximation (55) is well justified even for not-too-large values $\Delta y_{i j} / \ell_{0}$. For that we will calculate the dispersion laws of the renormalized fundamental EMPs using the exact expressions of $a_{i i}^{j j}\left(q_{x}, \Delta y_{i j}\right)$, i.e., with the double integral on the LHS of Eq. (55) evaluated numerically. In the latter case we will calculate $\omega_{j}^{\prime}$ from the standard expressions (59) -(61) of the appendix.

For a GaAs-based 2DEG and negligible dissipation the dispersion laws (51) and (52) for the renormalized, by the inter-LL Coulomb coupling, fundamental EMPs are shown in Fig. 1 by the solid curves. The parameters are $m^{*} \approx 6.1 \times 10^{-29} \mathrm{~g}, \epsilon \approx 12.5$, and $\Omega \approx 7.8 \times 10^{11} \mathrm{sec}^{-1}$ [19]. For $\nu=6$ and $B=3$ Tesla these parameters lead to $\hbar \omega_{c} \approx 5 \mathrm{meV}, \omega_{c} / \Omega \approx 10$. Here $\omega_{*}=2 e^{2} / \pi \hbar \epsilon \ell_{0}$ is a characteristic frequency almost equal, for the conditions stated, to $\omega_{c}$. We have also assumed $\Delta_{F 2}=\hbar \omega_{c} / 2$. This gives $v_{g 0} \approx \sqrt{5} \Omega \ell_{0}, v_{g 1} \approx \sqrt{3} \Omega \ell_{0}, v_{g 2} \approx \Omega \ell_{0}, \Delta y_{02} / \ell_{0} \approx\left(\omega_{c} / \Omega\right)[\sqrt{5}-1]$, 
$\Delta y_{01} / \ell_{0} \approx\left(\omega_{c} / \Omega\right)[\sqrt{5}-\sqrt{3}]$, and $\Delta y_{12} / \ell_{0} \approx\left(\omega_{c} / \Omega\right)[\sqrt{3}-1]$. The dashed curves show the fundamental EMPs of the totally decoupled $n=0$ (Eq. (20), topmost dashed curve), $n=1$, and $n=2$ (lowest dashed curve) LLs, i.e., the modes obtained by neglecting the inter-LL Coulomb coupling. In this case all coefficients $a_{i i}^{j j}$ on the RHS of Eqs. (47)-(49) vanish for $i \neq j$. This leads directly to the dispersion laws represented by the dashed curves in Fig. 1. The dotted curves show the dispersion laws of the renormalized fundamental EMPs using the exact expressions for $a_{i i}^{j j}\left(q_{x}, \Delta y_{i j}\right), i \neq j$. It is seen that each dotted curve is very close to the corresponding solid curve.

For the same parameters and conditions as in Fig. 1 we show, in Fig. $2, \rho\left(\omega, q_{x}, y\right) / \rho_{*}$ (solid and dotted curves) and $\rho\left(\omega, q_{x}, y\right) / 2 \rho_{*}$ (dashed curve) as a function of $Y=\left(y-y_{r 2}\right) / \ell_{0}$ with $\rho_{*}=\rho_{0}^{(0)}\left(\omega, q_{x}\right) / \sqrt{\pi} \ell_{0}$. The solid curve corresponds to the topmost solid curve in Fig. 1, Eq. (51), with $\rho_{1}^{(0)} / \rho_{0}^{(0)} \approx 1.0$ and $\rho_{2}^{(0)} / \rho_{0}^{(0)} \approx 1$. As can be seen the renormalized fundamental EMP of the $n=0$ LL has an acoustic spatial structure along the $y$ axis. The dashed curve corresponds to the lowest solid curve in Fig. 1, $\omega_{-}$of Eq. (52), with $\rho_{1}^{(0)} / \rho_{0}^{(0)} \approx-2.0$ and $\rho_{2}^{(0)} / \rho_{0}^{(0)} \approx 1$. Hence the renormalized fundamental EMP of the $n=2$ LL has an optical spatial structure though its dispersion law is purely acoustic. The dotted curve corresponds to the middle solid curve in Fig. 1, $\omega_{+}$of Eq. (52), with $\rho_{1}^{(0)} / \rho_{0}^{(0)} \approx-0.1$ and $\rho_{2}^{(0)} / \rho_{0}^{(0)} \approx-1.0$. Thus, the renormalized fundamental EMP of the $n=1$ LL has an optical spatial structure though its dispersion law is purely acoustic. Notice that the dependence of $\rho_{j}^{(0)} / \rho_{0}^{(0)}, j=1,2$, on $q_{x}$ is typically weak.

In Fig. 3 we use the same values for $m^{*}, \epsilon, \Omega$, and $\Delta_{F 2}=\hbar \omega_{c} / 2, \nu=6$ as in Fig. 1. However, we take $B=1.5$ Tesla and this leads to $\omega_{c} / \Omega \approx 5$. This gives $\sqrt{2}$ times larger values for $v_{g 0} \approx \sqrt{5} \Omega \ell_{0}, v_{g 1} \approx \sqrt{3} \Omega \ell_{0}$, and $v_{g 2} \approx \Omega \ell_{0}$, and twice smaller values for $\Delta y_{02} / \ell_{0} \approx\left(\omega_{c} / \Omega\right)[\sqrt{5}-1], \Delta y_{01} / \ell_{0} \approx\left(\omega_{c} / \Omega\right)[\sqrt{5}-\sqrt{3}]$, and $\Delta y_{12} / \ell_{0} \approx\left(\omega_{c} / \Omega\right)[\sqrt{3}-1]$ as compared with those in Fig. 1. All curves are marked as in Fig. 1 and show the corresponding dispersion laws.

In Fig. 4 we plot $\rho\left(\omega, q_{x}, y\right) / \rho_{*}$ as a function of $Y=\left(y-y_{r 2}\right) / \ell_{0}$ for the conditions corresponding to the solid curves in Fig. 3. All curves are marked as in Fig. 2. We have, respectively, $\rho_{1}^{(0)} / \rho_{0}^{(0)} \approx 1.0$ and $\rho_{2}^{(0)} / \rho_{0}^{(0)} \approx 1$ (solid curve), $\rho_{1}^{(0)} / \rho_{0}^{(0)} \approx-2.1$ and $\rho_{2}^{(0)} / \rho_{0}^{(0)} \approx 1.2$ (dashed curve), and $\rho_{1}^{(0)} / \rho_{0}^{(0)} \approx-0.1$ and $\rho_{2}^{(0)} / \rho_{0}^{(0)} \approx-1.0$ (dotted curve). As in Fg. 2, the renormalized fundamental EMP of the $n=0$ LL has an acoustic spatial structure along the $y$ axis while those of the $n=1$ and $n=2$ LL have an optical spatial structure though their dispersion laws are purely acoustic. As noted above, the dependence of $\rho_{j}^{(0)} / \rho_{0}^{(0)}, j=1,2$, on $q_{x}$ is typically weak.

\section{DISCUSSION AND CONCLUDING REMARKS}

We presented a fully microscopic model of EMPs in a RPA framework valid for integer $\nu \geq 1$ and confining potentials that are smooth on the $\ell_{0}$ scale but still sufficiently steep that LL flattening can be neglected 20. The model takes into account LL coupling and treats only very weak dissipation. The main results of the present work are as follows.

i) For moderately steep confining potential we presented a microscopic model that improves the quasi-microscopic approach of Refs. 11] and [18]. In particular, the model combines features of the following distinct edge wave mechanisms. In mostly classical models, e.g., [1], [7], the position of the edge does not vary but the charge density profile at the edge does. In a sence this edge wave mechanism is the analog of that for the Kelvin wave [21] at the edge of a rotating "shallow" sea with chirality determined by the Coriolis parameter which corresponds to the cyclotron frequency $\omega_{c}$. Another edge-wave mechanism, fully quantum mechanical, is that of Refs. [10], [17], and [22]- [23], in which, for $\nu=1$, only the edge position of an incompressible 2DEG of the lowest LL varies and with respect to which the density profile is that of the undisturbed 2DEG. The approach of these works is limited to the subspace of the lowest LL wave functions, neglects LL mixing and dissipation, and results in a single EMP with dispersion law similar to that in Ref. [1].

ii) We confirmed, for $\nu=1(2)$, the existence of EMP modes in addition to the fundamental EMP and single one obtained for vertically steep unperturbed electron density profile [1]. This is in line with our earlier quasi-microscopic results 11] for moderately steep [11] confining potentials. The additional modes result from an exact solution of Eq. (13) in terms of the complete set of the Hermite polynomials. With this expansion we can also make contact, for $\nu=1(2)$, with other microscopic theories [10], [17], and [22]- 223 that are limited to the subspace of the lowest LL wave functions. If we retain only the $n=0$ term in Eq. (13), we obtain only the $n=0$ LL fundamental mode with the same dispersion relation $\omega \sim q_{x} \ln q_{x}$. The additional modes result from retaining ( correspond to) higher-order terms in the expansion Eq. (13). This shows that the limitation to this LL subspace is too restrictive and indicates the importance of LL coupling even for $\nu=1(2)$.

iii) An important additional mode is the dipole EMP, presented in Sec. III B, with dispersion relation $\omega \sim q_{x}^{3}$. This differs markedly from the corresponding result $\omega \sim q_{x}$ of Ref. [11] and signals nonlocal responses that were previously neglected. This and other differences between the present results and those of Ref. [11] further indicate the importance of having a fully microscopic theory for EMPs. 
iv) As we showed, taking LL coupling into account is an essential ingredient especially for $\nu>2$. As Fig. 1 and Fig. 3 demonstrate, the coupling strongly renormalizes the uncoupled fundamental LL modes and results in one mode behaving as the usual one $\omega \sim q_{x} \ln q_{x}$ and the others behaving in an acoustic manner $\omega \sim q_{x}$. This partly reminds the results of Ref. [7] but the resemblance should not be overestimated because the two models are drastically different.

v) We have not treated spin effects beyond the HA or RPA. Our study is focused on important wave effects of non-spin nature and spin splitting is assumed negligible for even $\nu$. Thus, skyrmions and spin textures are not dealt with in this work. Though neglecting the spin splitting is a reasonable approximation, for even $\nu \geq 2$ in the bulk of the channel, its validity near the edges remains uncertain in view of the work of Refs. [15] and [24].

(vi) Finally, a few remarks are in order about the studies of Refs. [25] and [26] that used the RPA. The study of Ref. 26] is too simplified and in essence repeats the results of Ref. 25] and of works cited therein for the "optical" EMP. As for the results of Ref. [25], due to omitted important contributions, they are essentially different from ours for both the acoustic and the optical modes. As Sec. IV A shows, an important logarithmic term, caused by the Coulomb interaction, is missed in Eqs. (54a) and (54b) of Ref. 25]. In addition, Refs. 25] and [26] did not study a dipole mode or multipole modes pertaining to any occupied LL. These modes of ours are totally different than those of Secs. 3.1.6 and 3.1.7 of Ref. 25.

\section{ACKNOWLEDGMENTS}

This work was supported by NSERC Grant No. OGP0121756. In addition, O G B acknowledges partial support by the Ukrainian SFFI Grant No. 2.4/665.

\section{APPENDIX}

The expressions for the coefficients $a_{k}\left(q_{x}\right), k=1,2,3$ in Eq. (50) are as follows:

$$
\begin{gathered}
a_{2}\left(q_{x}\right)=-\left[\tilde{a}_{00}\left(q_{x}\right)+\tilde{a}_{11}^{11}\left(q_{x}, 0\right)+\tilde{a}_{22}^{22}\left(q_{x}, 0\right)\right] \\
a_{1}\left(q_{x}\right)=\tilde{a}_{00}\left(q_{x}\right) \tilde{a}_{11}^{11}\left(q_{x}, 0\right)+\tilde{a}_{00}\left(q_{x}\right) \tilde{a}_{22}^{22}\left(q_{x}, 0\right)+\tilde{a}_{11}^{11}\left(q_{x}, 0\right) \tilde{a}_{22}^{22}\left(q_{x}, 0\right)- \\
{\left[a_{00}^{22}\left(q_{x}, \Delta y_{02}\right)\right]^{2}-\left[a_{00}^{11}\left(q_{x}, \Delta y_{01}\right)\right]^{2}-\left[a_{11}^{22}\left(q_{x}, \Delta y_{12}\right)\right]^{2},}
\end{gathered}
$$

and

$$
\begin{aligned}
a_{0}\left(q_{x}\right)= & \tilde{a}_{00}\left(q_{x}\right)\left[a_{11}^{22}\left(q_{x}, \Delta y_{12}\right)\right]^{2}+\tilde{a}_{11}^{11}\left(q_{x}, 0\right)\left[a_{00}^{22}\left(q_{x}, \Delta y_{02}\right)\right]^{2}+\tilde{a}_{22}^{22}\left(q_{x}, 0\right)\left[a_{00}^{11}\left(q_{x}, \Delta y_{01}\right)\right]^{2}- \\
& \tilde{a}_{00}\left(q_{x}\right) \tilde{a}_{11}^{11}\left(q_{x}, 0\right) \tilde{a}_{22}^{22}\left(q_{x}, 0\right)-2 a_{00}^{11}\left(q_{x}, \Delta y_{01}\right) a_{11}^{22}\left(q_{x}, \Delta y_{12}\right) a_{00}^{22}\left(q_{x}, \Delta y_{02}\right) .
\end{aligned}
$$

Here $\tilde{a}_{00}\left(q_{x}\right)=a_{00}\left(q_{x}\right)+v_{g 0} /\left(2 e^{2} / \pi \hbar \epsilon\right)$, and $\tilde{a}_{i i}^{i i}\left(q_{x}, 0\right)=a_{i i}^{i i}\left(q_{x}, 0\right)+v_{g i} /\left(2 e^{2} / \pi \hbar \epsilon\right), i=1,2$. We also have $a_{22}^{22}\left(q_{x}, 0\right) \approx \ln \left(1 /\left|q_{x}\right| \ell_{0}\right)$ and, for $\Delta y_{02}, \Delta y_{12} \gg \ell_{0}, a_{00}^{22}\left(q_{x}, \Delta y_{02}\right) \approx \ln \left(1 /\left|q_{x}\right| \ell_{0}\right)-\ln \left(\Delta y_{02} / \ell_{0}\right)+0.1$, and $a_{11}^{22}\left(q_{x}, \Delta y_{12}\right) \approx \ln \left(1 /\left|q_{x}\right| \ell_{0}\right)-\ln \left(\Delta y_{12} / \ell_{0}\right)+0.1$

Eq. (50) corresponds to the irreducible case $Q\left(q_{x}\right)=q^{3}+r^{2}<0$, where $q=\left[a_{1}-a_{2}^{2} / 3\right] / 3, r=\left(a_{1} a_{2}-3 a_{0}\right) / 6-a_{2}^{3} / 27$. With $\rho=\sqrt{r^{2}-Q}$ and $\theta=\arctan (\sqrt{-Q} / r)$ the roots obey $\omega_{1}^{\prime}+\omega_{2}^{\prime}+\omega_{3}^{\prime}=-a_{2}$ and are given by

$$
\begin{gathered}
\omega_{1}^{\circ}=2 \rho^{1 / 3} \cos (\theta / 3)-a_{2} / 3, \\
\omega_{2}^{\circ}=-\rho^{1 / 3} \cos (\theta / 3)-a_{2} / 3-\sqrt{3} \rho^{1 / 3} \sin (\theta / 3),
\end{gathered}
$$

and

$$
\omega_{3}=-\rho^{1 / 3} \cos (\theta / 3)-a_{2} / 3+\sqrt{3} \rho^{1 / 3} \sin (\theta / 3)
$$


[1] V. A. Volkov and S. A. Mikhailov, Zh. Eksp. Teor. Fiz. 94, 217 (1988) [Sov. Phys. JETP 67, 1639 (1988)].

[2] M. Wassermeier, J. Oshinowo, J. P. Kotthaus, A. H. MacDonald, C. T. Foxon, and J. J. Harris, Phys. Rev. B 41, 10287 (1990).

[3] I. Grodnensky, D. Heitmann, and K. von Klitzing, Phys. Rev. Lett. 67, 1019 (1991).

[4] R. C. Ashoori, H. L. Stormer, L. N. Pfeiffer, K. W. Baldwin, and K. West, Phys. Rev. B 45, 3894 (1992); N. Balaban, Y. Meirav, H. Shtrikman, and V. Umansky, ibid 55, R13397 (1997).

[5] V. I. Tal'yanskii, A. V. Polisski, D. D. Arnone, M. Pepper, C. G. Smith, D. A. Ritchie, J. E. Frost, and G. A. C. Jones, Phys. Rev. B 46, 12427 (1992).

[6] N. B. Zhitenev, R. J. Haug, K. von Klitzing, and K. Eberl, Phys. Rev. Lett. 71, 2292 (1993); Phys. Rev. B 49, 7809 (1994).

[7] I. L. Aleiner and L. I. Glazman, Phys. Rev. Lett. 72, 2935 (1994).

[8] G. Ernst, R. J. Haug, J. Kuhl, K. von Klitzing, and K. Eberl, Phys. Rev. Lett. 77, 4245 (1996).

[9] J.. H. Han and D. J. Thouless, Phys. Rev. B 55, R1926 (1997).

[10] U. Zulicke, R. Bluhm, V. A. Kostelcky, and A. H. MacDonald, Phys. Rev. B 55, 9800 (1997).

[11] O. G. Balev and P. Vasilopoulos, Phys. Rev. B 56, 13252 (1997).

[12] O. G. Balev and P. Vasilopoulos, Phys. Rev. B 54, 4863 (1996).

[13] P. M. Platzmann and P. A. Wolff, Waves and Interactions in Solid State Plasmas, Solid State Physics, Eds. H. Ehrenreich, F. Seitz and D. Turnbull, Suppl. 13, Academic Press, Ney York and London (1973).

[14] O. G. Balev, Fiz. Tverd. Tela (Leningrad). 32, 871 (1990) [Sov. Phys. Solid State 32, 514 (1990)].

[15] O. G. Balev and P. Vasilopoulos, Phys. Rev. B 56, 6748 (1997).

[16] L. D. Landau and E. M. Lifshits, Electrodynamica of Continuous Media, Nauka, Moscow (1982).

[17] S. Giovanazzi, L. Pitaevskii, and S. Stringari, Phys. Rev. Lett. 72, 3230 (1994).

[18] O. G. Balev and P. Vasilopoulos, Phys. Rev. Lett., 81, 1481 (1998).

[19] G. Muller, D. Weiss, A. V. Khaetskii, K. von Klitzing, S. Koch, H. Nickel, W. Schlapp, and R. Losch, Phys. Rev. B 45, 3932 (1992).

[20] D. B. Chklovskii, B. I. Shklovskii, and L. I. Glazman, Phys. Rev. B 46, 4026 (1992); L. Brey, J. J. Palacios, and C. Tejedor, ibid 47,13884 (1993); T. Suzuki and Tsuneya Ando, J. Phys. Soc. Jpn. 62, 2986 (1993).

[21] Kelvin, William Thomson, Phil. Mag. x, 97 (1880).

[22] X. G. Wen, Int. J. Mod. Phys. B 6, 1711 (1992); M. Stone, H. W. Wyld, and R. L. Schult, Phys. Rev. B 45, 14156 (1992); C. de Chamon and X. G. Wen, ibid 49, 8227 (1994).

[23] J. H. Oaknin, L. Martin-Moreno, J. J. Palacios, and C. Tejedor, Phys. Rev. Lett. 74, 5120 (1995); J. J. Palacios and A. H. MacDonald, ibid 76, 118 (1996); U. Zulicke and A. H. MacDonald, Phys. Rev. B 54, 16813 (1996).

[24] V. Gudmundson and J. J. Palacios, Phys. Rev. B 52, 11266 (1995).

[25] V. A. Volkov and S. A. Mikhailov, in Modern Problems in Condensed Matter Sciences, ed. by V. M. Agranovich and A. A. Maradudin (North-Holland, Amsterdam, 1991), vol. 27.2, Chap. 15, p. 855.

[26] A. E. Kuchma and V. A. Sverdlov, Phys. Rev. B 54, 16333 (1996).

FIG. 1. Dispersion relation for $\nu=6$ and $B=3$ Tesla. The dashed curves are, from top to bottom, the decoupled fundamental modes of the $n=0, n=1$, and $n=2 \mathrm{LLs}$, respectively. The solid and dotted curves are for correspondingly the same coupled modes, $\omega_{*}=2 e^{2} / \pi \hbar \epsilon \ell_{0}$. For the solid curves approximation (Eq. (55)) is used.

FIG. 2. Spatial structure of the renormalized fundamental modes of the $n=0$ (solid curve), $n=1$ (dotted curve), and $n=2$ (dashed curve) LLs, whose dispersion laws are shown by the solid curves in Fig. 1. The solid and dotted curves show $\rho\left(\omega, q_{x}, y\right) / \rho_{*}$ and the dashed curve $\rho\left(\omega, q_{x}, y\right) / 2 \rho_{*}$ as a function of $Y=\left(y-y_{r 2}\right) / \ell_{0}$ with $\rho_{*}=\rho_{0}^{(0)}\left(\omega, q_{x}\right) / \sqrt{\pi} \ell_{0}$.

FIG. 3. Dispersion relation for $\nu=6$ and $B=1.5$ Tesla. All curves are marked as in Fig. 1.

FIG. 4. Spatial structure, $\rho\left(\omega, q_{x}, y\right) / \rho_{*}$, of the renormalized fundamental modes of the $n=0$ (solid curve), $n=1$ (dotted curve), and $n=2$ (dashed curve) LLs, whose dispersion laws are shown by the solid curves in Fig. 3. 


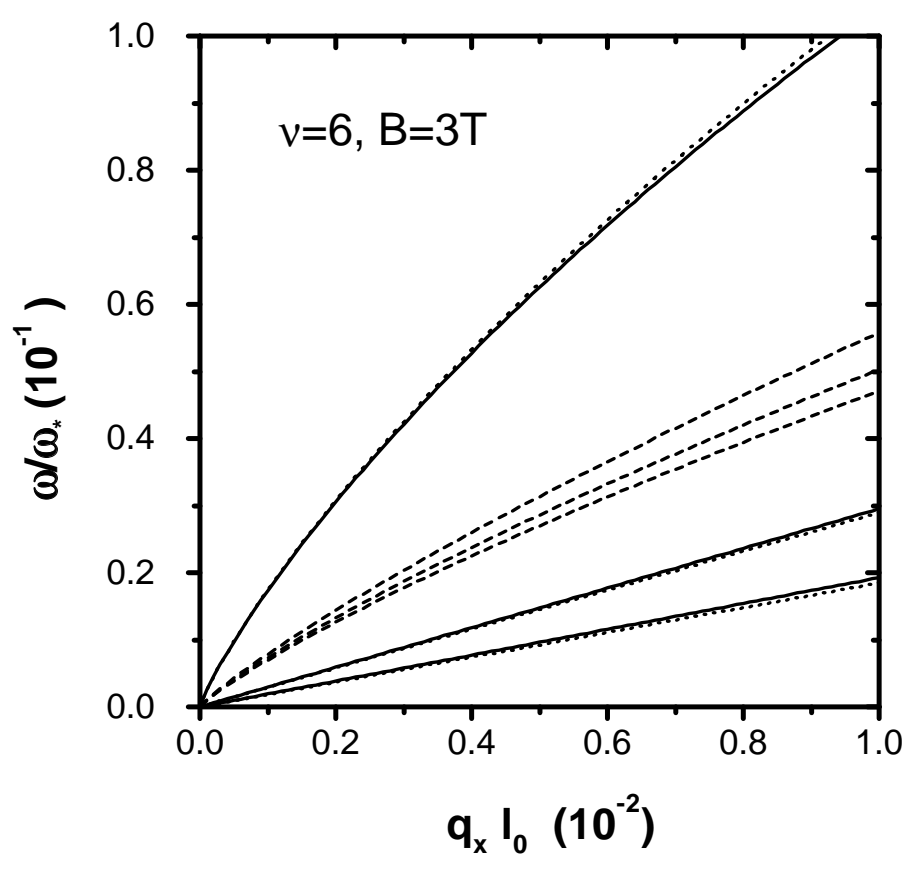

Fig. 1, Balev et al. 


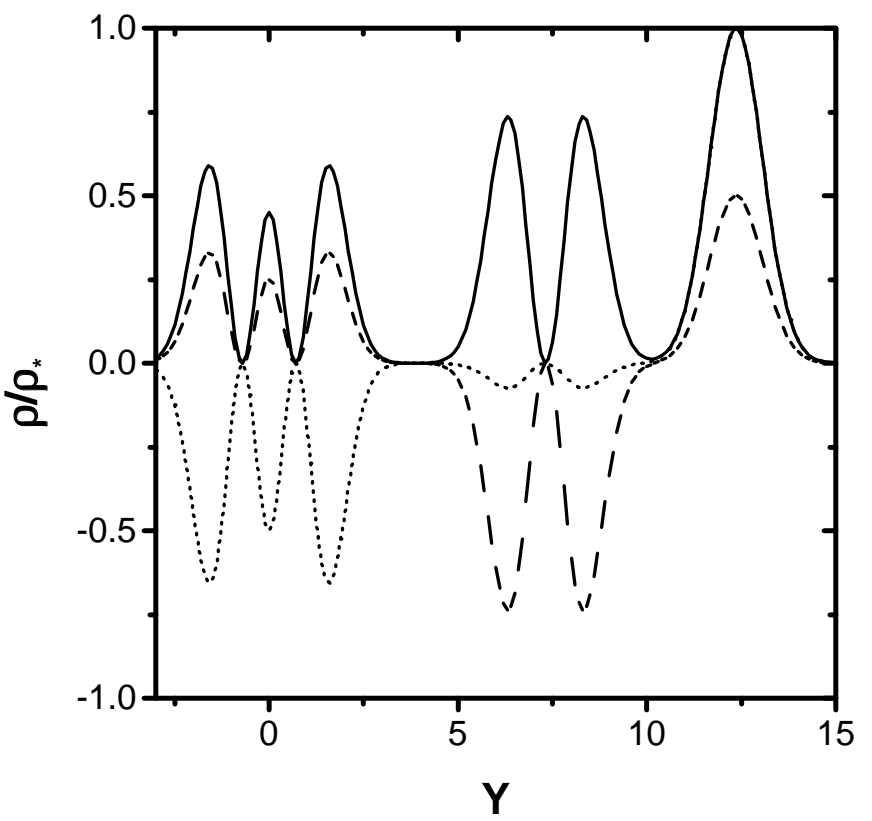

Fig. 2, Balev et al. 


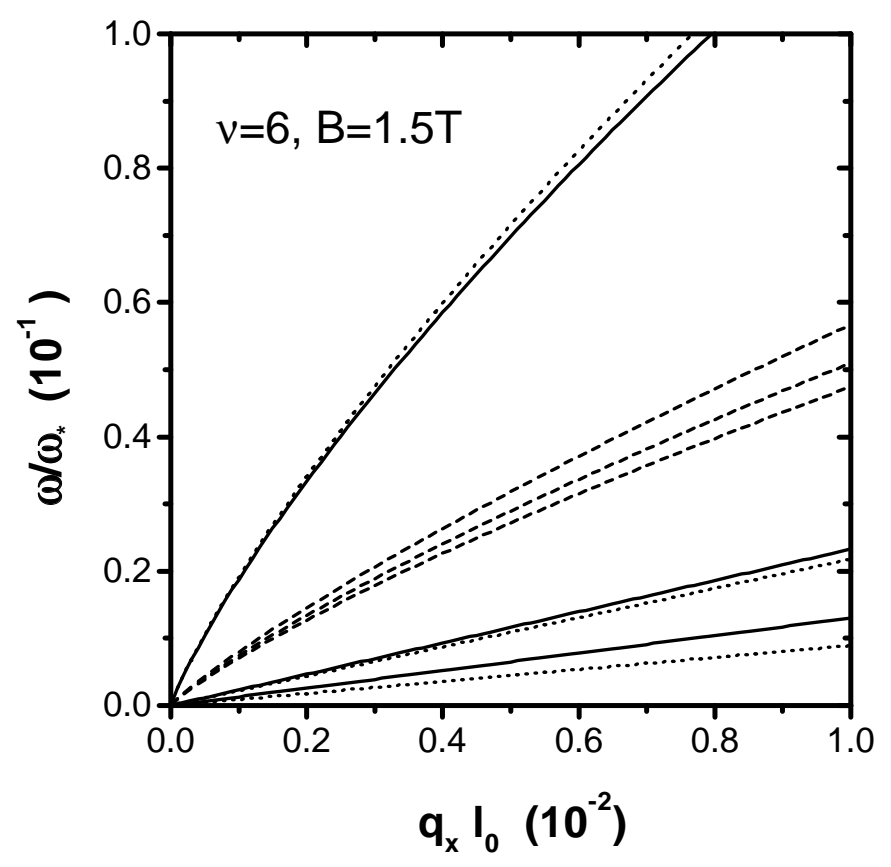

Fig. 3, Balev et al. 


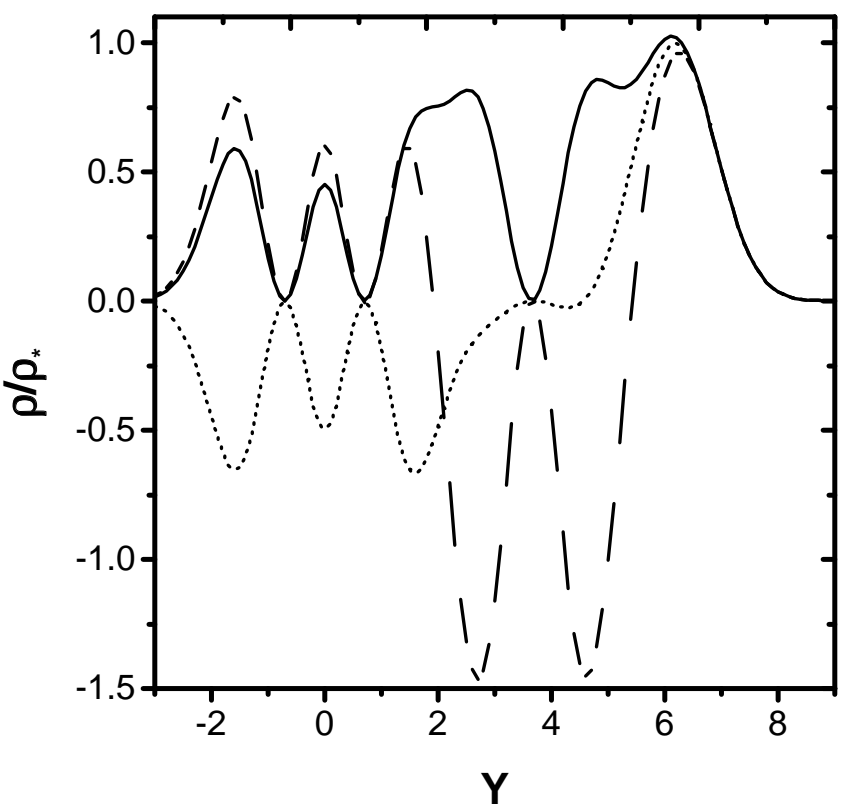

Fig. 4, Balev et al. 